\title{
Generalized Symmetries of Impulsive Gravitational Waves
}

\author{
Peter C. AICHELBURG 周 \\ Institut für Theoretische Physik, Universität Wien \\ Boltzmanngasse 5, A - 1090 Wien, AUSTRIA \\ and \\ Herbert BALASIN 尚尚 \\ Institut für Theoretische Physik, Technische Universität Wien \\ Wiedner Hauptstraße 8-10, A - 1040 Wien, AUSTRIA
}

Dedication: We feel honored to dedicate this article to Andrzej Trautman on the occasion of his $8^{2}$-th birthday

\begin{abstract}
We generalize previous [1] work on the classification of $\left(C^{\infty}\right)$ symmetries of plane-fronted waves with an impulsive profile. Due to the specific form of the profile it is possible to extend the group of normalform-preserving diffeomorphisms to include non-smooth transformations. This extension entails a richer structure of the symmetry algebra generated by the (non-smooth) Killing vectors.

PACS numbers: 9760L, 0250

UWThPh $-1996-28$
TUW $96-07$
April 1996
\end{abstract}

* e-mail: pcaich @ pap.univie.ac.at

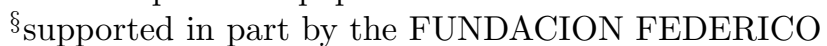

$\dagger$ e-mail: hbalasin @ email.tuwien.ac.at

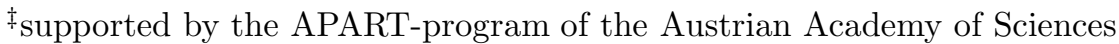




\section{Introduction}

In a previous paper [1] the authors considered symmetries of impulsive planefronted waves with parallel rays. The motivation for this study was the failure of the classical work by Jordan, Ehlers and Kundt (JEK) [2] for wave profiles which are generalized functions [3]. In order to solve this problem a new systematic approach for the classification of general pp-waves was presented [1], which was in the following applied to the simplest class of impulsive spacetimes, where the wave is concentrated on a single null hyperplane. It was shown that even vacuum spacetimes within this class contain new symmetries which have no non-impulsive analogue.

In the above mentioned analysis it was assumed that the symmetry generating vector fields are smooth, because only then it is guaranteed that in general Killing's equation is well-defined. In present paper we relax this condition, i.e. we do not require $C^{\infty}$-Killing fields from the outset, but rather adapt to the profile of the wave under consideration. What we require is that for a given profile, each term in Killing's equation is well defined in the sense of generalized functions. Again we apply this reasoning to our prototype of impulsive waves. The analysis shows that in this class there exist, besides of the smooth Killing fields found in [1], symmetries that are only continuous or even discontinuous. We give a complete classification for these spacetimes in terms of their symmetries and show that the structure of these "generalized symmetries" is much richer. As in [1] we do not impose Einsteins vacuum equation.

It was argued by R. Penrose [国] that although the impulsive waves have a delta-like metric component in adapted coordinates, the metric can be made continuous by going over to the so-called Rosen form [5]. This of course involves a discontinuous transformation by which the originally smooth Killing fields become non-smooth. We show this explicitly for impulsive plane waves, which admit a five parameter group of isometries.

The paper is organized as follows: in section 1 we introduce pp-wave spacetimes in adapted (normal form) coordinates. We generalize the so-called normal-form-preserving diffeomorphisms to non-smooth ones for waves with impulsive profile. In section 2 we investigate the abstract group structure of these transformations and classify its Lie-algebra in terms of adjoint orbits. Then we impose the "canonical" representative of each orbit as generator of an isometry and obtain the corresponding wave profile. Since, as pointed out in [1], any impulsive wave admits a three parameter group of isometries, 
this method leads to waves with at least four Killing vectors. In section 3 spacetimes belonging to even higher symmetry classes are obtained from the combination of different generators. This is a non-trivial procedure since in general the generators cannot be transformed to their canonical form simultaneously. The complete classification is presented in a table at the end of this section. Finally, in section 4 we transform the plane impulsive wave to the Rosen form.

\section{1) Impulsive pp-waves}

In this section we briefly recall the definition of pp-waves, especially impulsive ones. With respect to adapted coordinates the metric takes the "normal" form [6]

$$
d s^{2}=-d u d v+d x^{2}+f(u, x) d u^{2},
$$

where $x$ are flat coordinates in the two-dimensional spacelike subspace $u=$ $v=$ const. Therefore a pp-wave is completely characterized by its profilefunction $f(u, x)$, which is unique up to normal form (nfp) preserving diffeomorphisms [2, 1]. The latter are given by

$$
\begin{aligned}
\tilde{u} & =a u+b, \\
\tilde{v} & =\frac{1}{a}\left(v+2\left(\Omega x \cdot d^{\prime}(u)+n(u)\right)\right), \\
\tilde{x} & =\Omega x+d(u) .
\end{aligned}
$$

Here we follow the notation introduced in [1] where $a$ and $b$ are constants, $\Omega$ is in $S O(2)$ and $d(u)$ and $n(u)$ denote a two-dimensional vector field and a function respectively. Impulsive pp-waves are further restricted by requiring their curvature to be concentrated on the null hyperplane $u=0$. This implies that the profile is of the form

$$
f(u, x)=f(x) \delta(u),
$$

where $f(x)$ will in the following, by a slight abuse of language, also be referred to as the profile.

For a profile that is genuinely distributional in nature only $C^{\infty}$ nfpdiffeomorphisms are allowed. However, taking into account that $\delta(u)$ is an order zero distribution it is possible to extend the class of nfp-transformations 
by including terms which are non-smooth at $u=0$. Let us therefore examine the transformation behavior of the profile with respect to a nfptransformation

$$
\begin{aligned}
\tilde{f}(u, x) & =a^{2} \delta(a u) f(\Omega x+d(u))+d^{\prime 2}(u)-2\left(d^{\prime \prime}(u) \cdot \Omega x+n^{\prime}(u)\right) \\
& =a \delta(u) f(\Omega x+d(0))+d^{\prime 2}(u)-2\left(d^{\prime \prime}(u) \cdot \Omega x+n^{\prime}(u)\right),
\end{aligned}
$$

where we have set the parameter $b=0$ since its sole effect amounts in a shift of the location of the pulse. In order to produce at most delta-function terms the non-smooth generalization has to be of the form

$$
\begin{aligned}
& d(u)=d_{s}(u)+\theta(u) d_{+}(u), \\
& n(u)=n_{s}(u)+\theta(u) n_{+}(u),
\end{aligned} \quad d_{s}, d_{+}, n_{s}, n_{+} \in C^{\infty},
$$

where $\theta(u)$ is the Heaviside step function. The second line of (1) tells us that $d(u)$ has to be well defined at $u=0$ thus requiring $d_{+}(0)=0$. As a consequence the transformed profile becomes

$$
\begin{aligned}
\tilde{f}(u, x) & =\delta(u)\left(a f\left(\Omega x+d_{0}\right)-2\left(d_{1}^{+} \cdot \Omega x+n_{0}^{+}\right)\right) \\
& +\theta(u)\left(d_{+}^{\prime 2}(u)+2 d_{s}^{\prime}(u) \cdot d_{+}^{\prime}(u)-2\left(d_{+}^{\prime \prime}(u) \cdot \Omega x+n_{+}^{\prime}(u)\right)\right) \\
& +\left(d_{s}^{\prime 2}(u)-2\left(d_{s}^{\prime \prime}(u) \cdot \Omega x+n_{s}^{\prime}(u)\right)\right),
\end{aligned}
$$

where we adopted the following notation: indices 0 and 1 denote the value of the function and its derivative at $u=0$ respectively. ¿From $\tilde{f}(u, x)=$ $\tilde{f}(x) \delta(u)$ and since the smooth part of (5) has to vanish separately, we find

$$
\begin{aligned}
d_{s}^{\prime \prime}(u)=0 & \Longrightarrow d_{s}=d_{0}+u d_{1} \\
n_{s}^{\prime}(u)=\frac{1}{2}{d_{s}^{\prime}}^{2}(u) & \Longrightarrow n_{s}(u)=n_{0}+\frac{u}{2} d_{1}^{2} .
\end{aligned}
$$

The remaining terms may be split into those contributing for positive values of $u$ and those which are concentrated at $u=0$.

$$
\begin{aligned}
& d_{+}^{\prime \prime}(u)=0 \Longrightarrow d_{+}(u)=d_{1}^{+} u \\
& n_{+}^{\prime}(u)=d_{1} \cdot d_{1}^{+}+\frac{1}{2} d_{1}^{+2} \Longrightarrow n_{+}(u)=n_{0}^{+}+\frac{u}{2}\left(d_{1}^{+2}+2 d_{1} \cdot d_{1}^{+}\right) .
\end{aligned}
$$

Finally, we obtain from (5) the transformation law for $f(x)$ and the form of $d(u)$ and $n(u)$

$$
\tilde{f}(x)=a f\left(\Omega x+d_{0}\right)-2\left(d_{1}^{+} \cdot \Omega x+n_{0}^{+}\right)
$$




$$
\begin{aligned}
& d(u)=d_{0}+u d_{1}+\theta(u) u d_{1}^{+} \\
& n(u)=n_{0}+\frac{u}{2} d_{1}^{2}+\theta(u)\left(n_{0}^{+}+\frac{u}{2}\left(d_{1}^{+2}+2 d_{1} \cdot d_{1}^{+}\right)\right) .
\end{aligned}
$$

This extends the restricted nfp-subgroup ( $\mathrm{rnfp}$ ) found in [1] of smooth nfptransformations, preserving (3) by three additional parameters $\left(n_{0}^{+}, d_{1}^{+}\right)$.

\section{2) Non-smooth rnfp-group and adjoint orbits}

The non-smooth rnfp transformations form a finite-dimensional group whose elements $g$ are parametrized by

$$
g=\left(a, \Omega ; d_{0}, d_{1}, d_{1}^{+} ; n_{0}, n_{0}^{+}\right),
$$

which in turn gives rise to the parameterization of the multiplication map $g_{3}=g_{2} g_{1}$

$$
\begin{aligned}
a_{3} & =a_{2} a_{1} \\
\Omega_{3} & =\Omega_{2} \Omega_{1} \\
d_{03} & =\Omega_{2} d_{01}+d_{02} \\
d_{13} & =\Omega_{2} d_{11}+a_{1} d_{12} \\
d_{13}^{+} & =\Omega_{2} d_{11}^{+}+a_{1} d_{12}^{+} \\
n_{03} & =n_{01}+a_{1} n_{02}+a_{1} \Omega_{2} d_{01} \cdot d_{12} \\
n_{03}^{+} & =n_{01}^{+}+a_{1} n_{02}^{+}+a_{1} \Omega_{2} d_{01} \cdot d_{12}^{+} .
\end{aligned}
$$

The second index $i=1,2,3$ in $d$ and $n$ refers to the group element $g_{i}$ to which it is associated. The physical equivalence of profiles under rnfptransformations is reflected in the equivalence of Killing vectors under the corresponding action of the rnfp-group. At the level of the group this amounts to the adjoint action of the non-smooth rnfp-group on its Lie algebra. ¿From (10) it is easy to obtain the adjoint action of an arbitrary group element element $g$ on the Lie-algebra of the non-smooth rnfp-group, whose general element $X$ will be parametrized by

$$
X=\left(\alpha, \omega ; D_{0}, D_{1}, D_{1}^{+} ; N_{0}, N_{0}^{+}\right) .
$$

$$
\tilde{\alpha}=\alpha \quad \tilde{X}=\operatorname{Ad}(g) X
$$




$$
\begin{aligned}
\tilde{\omega} & =\omega \\
\tilde{D}_{0} & =\Omega D_{0}-\omega d_{0} \\
\tilde{D}_{1} & =\frac{1}{a}\left(\Omega D_{1}-\omega d_{1}+\alpha d_{1}\right) \\
\tilde{D}_{1}^{+} & =\frac{1}{a}\left(\Omega D_{1}^{+}-\omega d_{1}^{+}+\alpha d_{1}^{+}\right) \\
\tilde{N}_{0} & =\frac{1}{a}\left(N_{0}+\alpha n_{0}+\Omega D_{0} \cdot d_{1}-d_{0} \cdot \Omega D_{1}-\alpha d_{0} \cdot d_{1}-\omega d_{0} \cdot d_{1}\right) \\
\tilde{N}_{0}^{+} & =\frac{1}{a}\left(N_{0}^{+}+\alpha n_{0}^{+}+\Omega D_{0} \cdot d_{1}^{+}-d_{0} \cdot \Omega D_{1}^{+}-\alpha d_{0} \cdot d_{1}^{+}-\omega d_{0} \cdot d_{1}^{+}\right)
\end{aligned}
$$

The infinitesimal form of the transformation law of the profile is obtained from (8) by considering $g$ to be an arbitrary curve through the unity with tangent $X$

$$
\delta f=\alpha f-x \omega \partial f+\left(D_{0} \partial\right) f-2\left(D_{1}^{+} x+N_{0}^{+}\right) .
$$

If in addition we require $X$ to be Killing the variation $\delta f$ of the profile has to vanish. Since neither $D_{1}$ nor $N_{0}$ appear in (13) they are immediately recognized as Killing parameters thereby giving rise to a three-dimensional Killing algebra for the generic profile $f(x)$. Moreover, this three-dimensional subalgebra forms an abelian ideal within the Lie algebra of non-smooth rnfp transformations. Consequently only the corresponding quotient algebra, whose general element may be characterized by setting $D_{1}$ and $N_{0}$ to zero in (11), acts effectively on the space of profiles. Therefore the problem of characterizing the adjoint orbits of the full group in the full algebra is reduced to the corresponding subgroup and subalgebra respectively. Explicitly this procedure results in

$$
\begin{aligned}
\tilde{\alpha} & =\alpha \\
\tilde{\omega} & =\omega \\
\tilde{D}_{0} & =\Omega D_{0}-\omega d_{0} \\
\tilde{D}_{1}^{+} & =\frac{1}{a}\left(\Omega D_{1}^{+}-\omega d_{1}^{+}+\alpha d_{1}^{+}\right) \\
\tilde{N}_{0}^{+} & =\frac{1}{a}\left(N_{0}^{+}+\alpha n_{0}^{+}+\Omega D_{0} \cdot d_{1}^{+}-\Omega D_{1}^{+} \cdot d_{0}-\alpha d_{0} \cdot d_{1}^{+}-\omega d_{0} \cdot d_{1}^{+}\right),
\end{aligned}
$$

which gives rise to the following classification of adjoint orbits:

1) $\underline{\omega \neq 0, \alpha \neq 0}$ 


$$
\begin{aligned}
& X=\left(1, \omega_{1} ; 0,0,0 ; 0,0\right) \\
& \operatorname{Stab}(X)=\{(a, \Omega ; 0,0,0 ; 0,0)\} \\
& X=\left(0, \omega_{1} ; 0,0,0 ; 0,1\right) \\
& \operatorname{Stab}(X)=\left\{\left(1, \Omega ; 0,0,0 ; 0, n_{0}^{+}\right)\right\} \\
& \text {3) } \frac{\omega \neq 0, \alpha=0, N_{0}^{+}=0}{X=\left(0, \omega_{1} ; 0,0,0 ; 0,0\right)} \\
& \operatorname{Stab}(X)=\left\{\left(a, \Omega ; 0,0,0 ; 0, n_{0}^{+}\right)\right\} \\
& X=\left(1,0 ; D_{0}, 0,0 ; 0,0\right) \\
& \operatorname{Stab}(X)=\left\{\left(a, i d ; d_{0}, 0,0 ; 0,0\right)\right\} \\
& X=(1,0 ; 0,0,0 ; 0,0)
\end{aligned}
$$

$\operatorname{Stab}(X)=\left\{\left(a, i d ; d_{0}, 0, d_{1}^{+} ; 0, n_{0}^{+}\right)\right\} \quad$ with $D_{0} \cdot d_{1}^{+}=D_{1}^{+} \cdot d_{0}$

7) $\underline{\omega}=0, \alpha=0, D_{0} \neq 0, D_{1}^{+}=0$

$$
X=\left(0,0 ; D_{0}, 0,0 ; 0,0\right)
$$

$\operatorname{Stab}(X)=\left\{\left(a, i d ; d_{0}, 0, d_{1}^{+} ; 0, n_{0}^{+}\right)\right\} \quad$ with $D_{0} \cdot d_{1}^{+}=0$

8) $\underline{\omega}=0, \alpha=0, D_{0}=0, D_{1}^{+} \neq 0$

$X=\left(0,0 ; 0, D_{1}^{+}, 0 ; 0,0\right)$

$\operatorname{Stab}(X)=\left\{\left(a, i d ; d_{0}, 0, d_{1}^{+} ; 0, n_{0}^{+}\right)\right\} \quad$ with $D_{1}^{+} \cdot d_{0}=0$

9) $\underline{\omega}=0, \alpha=0, D_{0}=0, D_{1}^{+}=0, N_{0}^{+} \neq 0$

$X=(0,0 ; 0,0,0 ; 0,1)$

$\operatorname{Stab}(X)=\left\{\left(a, \Omega ; d_{0}, 0, d_{1}^{+} ; 0, n_{0}^{+}\right)\right\}$, 
where $X$ and $\operatorname{Stab}(X)$ denote the canonical representative of the orbit and its stability group respectively.

\section{3) Profiles with four and more Killing vectors}

The task of finding profiles with a four-parameter symmetry group is now simply reduced to inserting the vectors $X$ into Killing's equation which determines the corresponding profile $f$.

1) $0=f-x \omega_{1} \partial f, \quad f=\gamma_{1} \partial_{\phi} f$

$$
f=h(\rho) e^{\frac{1}{\gamma_{1}} \phi}
$$

2) $0=-x \omega_{1} \partial f-2, \quad \gamma_{1} \partial_{\phi} f=-2$

$$
f=h(\rho)-\frac{2}{\gamma_{1}} \phi
$$

3) $0=-x \omega_{1} \partial f, \quad \partial_{\phi} f=0$

$$
f=h(\rho)
$$

4) $f+\left(D_{0} \partial\right) f=0$

$$
f=h\left(\tilde{D}_{0} x\right) \exp \left(-\frac{D_{0} x}{D_{0}^{2}}\right)
$$

5) $f=0 \Longrightarrow$ flat space

6) $\left(D_{0} \partial\right) f-2 D_{1}^{+} x=0$

$$
f=h\left(\tilde{D}_{0} x\right)+\frac{1}{\left(D_{0}^{2}\right)^{2}}\left[\left(D_{1}^{+} D_{0}\right)\left(D_{0} x\right)^{2}+2\left(D_{1}^{+} \tilde{D}_{0}\right)\left(\tilde{D}_{0} x\right)\left(D_{0} x\right)\right]
$$

7) $\left(D_{0} \partial\right) f=0$

$$
f=h\left(\tilde{D}_{0} x\right)
$$

8) $-2 D_{1}^{+} x=0 \Longrightarrow D_{1}^{+}=0 \Longrightarrow$ no solution

9) $-2 N_{0}^{+}=0 \Longrightarrow N_{0}^{+}=0 \Longrightarrow$ no solution. 
In cases 1) and 2) $\rho$ and $\phi$ denote circular polar coordinates. ¿From the above table we see that the relevant cases with four Killing vectors are 1) to 4) and 6) and 7).

In order to obtain classes with even higher symmetries we have to combine the above cases, by imposing additional Killing vectors. This, however, cannot be done by simply taking $X$ from the classes 1 ) to 9 ), since they may not attain their "canonical" form in the same system of coordinates. Suppose we would like to find spacetimes with symmetries from orbit 1 and orbit 2 . We may start with the canonical representative $X_{1}$ from orbit 1 , but then we have to take the generic representative from orbit 2, i.e. $g X_{2} g^{-1}$. However, since the stability groups do not change the correponding canonical form, we may use, without loss in generality, $\hat{g} X_{2} \hat{g}^{-1}$, where $\hat{g}=g_{1} g g_{2}$, with $g_{1}$ and $g_{2}$ belonging to the respective stability groups of $X_{1}$ and $X_{2}$. We then impose $\hat{g} X_{2} \hat{g}^{-1}$ to be Killing thereby restricting the profile found for $X_{1}$ further. This will be done for all combinations. The $\hat{g}$ obtained by combining elements from two orbits are:

$$
\begin{aligned}
& 1+2 g_{1} g g_{2}=\left(a_{1} a, \Omega_{1} \Omega \Omega_{2} ; \Omega_{1} d_{0}, 0, \Omega_{1} d_{1}^{+} ; 0, n_{02}^{+}+n_{0}^{+}\right) \\
& \hat{g}=\left(1, i d ; d_{0}, 0, d_{1}^{+} ; 0,0\right) \\
& 1+3 g_{1} g g_{3}=\left(a_{1} a a_{3}, \Omega_{1} \Omega \Omega_{3} ; \Omega_{1} d_{0}, 0, a_{3} \Omega_{1} d_{1}^{+} ; 0, n_{03}^{+}+a_{3} n_{0}^{+}\right) \\
& \hat{g}=\left(1, i d ; d_{0}, 0, d_{1}^{+} ; 0,0\right) \\
& 1+4 g_{1} g g_{4}=\left(a_{1} a a_{4}, \Omega_{1} \Omega ; \Omega_{1}\left(\Omega d_{04}+d_{0}\right), 0, a_{4} \Omega_{1} d_{1}^{+} ; 0, a_{4} n_{0}^{+}+a_{4} d_{1}^{+} \cdot \Omega d_{04}\right) \\
& \hat{g}=\left(1, i d ; 0,0, \Omega^{-1} d_{1}^{+} ; 0, n_{0}^{+},-d_{0}^{2}\right) \\
& 1+6 g_{1} g g_{6}=\left(a_{1} a a_{6}, \Omega_{1} \Omega ; \Omega_{1}\left(\Omega d_{06}+d_{0}\right), 0, \Omega_{1}\left(\Omega d_{16}+a_{6} d_{1}^{+}\right) ; 0, n_{06}^{+}+a_{6} n_{0}^{+}+\right. \\
& \left.a_{6} d_{1}^{+} \cdot \Omega d_{06}\right) \quad D_{06} \cdot d_{16}^{+}=D_{16}^{+} \cdot d_{06} \\
& \hat{g}=\left(1, i d ; 0,0, d_{16}^{+}+\Omega^{-1} d_{1}^{+} ; 0,0\right) \text { with } \tilde{D}_{06}\left(d_{16}^{+}+\Omega^{-1} d_{1}^{+}\right)=0 \\
& 1+7 g_{1} g g_{7}=\left(a_{1} a a_{7}, \Omega_{1} \Omega ; \Omega_{1}\left(\Omega d_{07}+d_{0}\right), 0, \Omega_{1}\left(\Omega d_{17}+a_{7} d_{1}^{+}\right) ; 0, n_{07}^{+}+a_{7} n_{0}^{+}+\right. \\
& \left.a_{7} d_{1}^{+} \cdot \Omega d_{07}\right) \quad D_{07} \cdot d_{17}^{+}=0 \\
& \hat{g}=\left(1, i d ; 0,0, d_{17}^{+}+\Omega^{-1} d_{1}^{+} ; 0,0\right) \text { with } \tilde{D}_{07}\left(d_{17}^{+}+\Omega^{-1} d_{1}^{+}\right)=0
\end{aligned}
$$




$$
\begin{aligned}
& 2+3 g_{2} g g_{3}=\left(a a_{3}, \Omega_{2} \Omega \Omega_{3} ; \Omega_{2} d_{0}, 0, a_{3} \Omega_{2} d_{1}^{+} ; 0, n_{03}^{+}+a_{3} n_{0}^{+}+a_{3} a n_{02}^{+}\right) \\
& \hat{g}=\left(1, i d ; d_{0}, 0, \frac{1}{a} d_{1}^{+} ; 0,0\right) \\
& 2+4 g_{2} g g_{4}=\left(a a_{4}, \Omega_{2} \Omega ; \Omega_{2}\left(\Omega d_{04}+d_{0}\right), 0, a_{4} \Omega_{2} d_{1}^{+} ; 0, a_{4}\left(n_{0}^{+}+a n_{02}^{+}\right)+a_{4} \Omega d_{04}\right. \text {. } \\
& \left.d_{1}^{+}\right) \\
& \hat{g}=\left(1, i d ; 0,0, \frac{1}{a} \Omega^{-1} d_{1}^{+} ; 0,0\right) \\
& 2+6 g_{2} g g_{6}=\left(a a_{6}, \Omega_{2} \Omega ; \Omega_{2} \Omega d_{06}+\Omega_{2} d_{0}, 0, \Omega_{2} \Omega d_{16}^{+}+a_{6} \Omega_{2} d_{1}^{+} ; 0, n_{06}^{+}+\right. \\
& \left.a_{6}\left(n_{0}^{+}+a n_{02}^{+}\right)+a_{6} \Omega d_{06} \cdot d_{1}^{+}\right) \quad D_{06} \cdot d_{16}^{+}=D_{16}^{+} \cdot d_{06} \\
& \hat{g}=\left(1, i d ; 0,0, d_{16}^{+}+\frac{1}{a} \Omega^{-1} d_{1}^{+} ; 0,0\right) \text { with } \tilde{D}_{06}\left(d_{16}^{+}+\frac{1}{a} \Omega^{-1} d_{1}^{+}\right)=0 \\
& 2+7 g_{2} g g_{7}=\left(a a_{7}, \Omega_{2} \Omega ; \Omega_{2} \Omega d_{07}+\Omega_{2} d_{0}, 0, \Omega_{2} \Omega d_{17}^{+}+a_{7} \Omega_{2} d_{1}^{+} ; 0\right. \text {, } \\
& \left.n_{07}^{+}+a_{7}\left(n_{0}^{+}+a n_{02}^{+}\right)+a_{7} \Omega d_{07} \cdot d_{1}^{+}\right) \quad D_{07} \cdot d_{17}^{+}=0 \\
& \hat{g}=\left(1, i d ; 0,0, d_{17}^{+}+\frac{1}{a} \Omega^{-1} d_{1}^{+} ; 0,0\right) \text { with } \tilde{D}_{07}\left(d_{17}^{+}+\frac{1}{a} \Omega^{-1} d_{1}^{+}\right)=0 \\
& 3+4 g_{2} g g_{4}=\left(a_{3} a a_{4}, \Omega_{3} \Omega ; \Omega_{3} d_{0}+\Omega_{3} \Omega d_{04}, 0, a_{4} \Omega_{3} d_{1}^{+} ; 0, a_{4}\left(n_{0}^{+}+a n_{03}^{+}\right)\right) \\
& \hat{g}=\left(1, i d ; 0,0, \Omega^{-1} d_{1}^{+} ; 0,0\right) \\
& 3+6 g_{3} g g_{6}=\left(a_{3} a a_{6}, \Omega_{3} \Omega ; \Omega_{3} \Omega d_{06}+\Omega_{3} d_{0}, 0, \Omega_{3} \Omega d_{16}^{+}+a_{6} \Omega_{3} d_{1}^{+} ; 0\right. \text {, } \\
& \left.n_{06}^{+}+a_{6}\left(n_{0}^{+}+a n_{03}^{+}\right)+a_{6} \Omega d_{06} \cdot d_{1}^{+}\right) \quad D_{06} \cdot d_{16}^{+}=D_{16}^{+} \cdot d_{06} \\
& \hat{g}=\left(1, i d ; 0,0, d_{16}^{+}+\Omega^{-1} d_{1}^{+} ; 0,0\right) \text { with } \tilde{D}_{06}\left(d_{16}^{+}+\Omega^{-1} d_{1}^{+}\right)=0 \\
& 3+7 g_{3} g g_{7}=\left(a_{3} a a_{7}, \Omega_{3} \Omega ; \Omega_{3} \Omega d_{07}+\Omega_{3} d_{0}, 0, \Omega_{3} \Omega d_{17}^{+}+a_{7} \Omega_{3} d_{1}^{+} ; 0\right. \text {, } \\
& \left.n_{07}^{+}+a_{7}\left(n_{0}^{+}+a n_{03}^{+}\right)+a_{7} \Omega d_{07} \cdot d_{1}^{+}\right) \quad D_{07} \cdot d_{17}^{+}=0 \\
& \hat{g}=\left(1, i d ; 0,0, d_{17}^{+}+\Omega^{-1} d_{1}^{+} ; 0,0\right) \text { with } \tilde{D}_{07}\left(d_{17}^{+}+\Omega^{-1} d_{1}^{+}\right)=0 \\
& 4+6 g_{4} g g_{6}=\left(a_{4} a a_{6}, \Omega ; \Omega d_{06}+d_{0}+d_{04}, 0, \Omega d_{16}^{+}+a_{6} d_{1}^{+} ; 0, n_{06}^{+}+a_{6} n_{0}^{+}+a_{6} \Omega d_{06}\right. \text {. } \\
& \left.d_{1}^{+}\right) \quad D_{06} \cdot d_{16}^{+}=D_{16}^{+} \cdot d_{06} \\
& \hat{g}=(1, \Omega ; 0,0,0 ; 0,0) \\
& 4+7 g_{4} g g_{7}=\left(a_{4} a a_{7}, \Omega ; \Omega d_{07}+d_{0}+d_{04}, 0, \Omega d_{17}^{+}+a_{7} d_{1}^{+} ; 0, n_{07}^{+}+a_{7} n_{0}^{+}+a_{7} \Omega d_{07}\right. \text {. } \\
& \left.d_{1}^{+}\right) \quad D_{07} \cdot d_{17}^{+}=0 \\
& \hat{g}=\left(1, i d ; 0,0, \Omega\left(d_{17}^{+}+\Omega^{-1} d_{1}^{+}\right) ; 0,0\right) \text { with } \Omega^{-1} \tilde{D}_{07}\left(d_{17}^{+}+\Omega^{-1} d_{1}^{+}\right)=0
\end{aligned}
$$




$$
\begin{aligned}
& 6+7 g_{6} g g_{7}=\left(a_{6} a a_{7}, \Omega ; \Omega d_{07}+d_{0}+d_{06}, 0, \Omega d_{17}^{+}+a_{7} d_{1}^{+}+a a_{7} d_{16}^{+} ; 0\right. \text {, } \\
& \left.n_{07}^{+}+a_{7}\left(n_{0}^{+}+a n_{06}^{+}\right)+a d_{0} \cdot d_{16}^{+} a_{7} \Omega d_{07} \cdot\left(d_{1}^{+} a d_{16}^{+}\right)\right) \\
& D_{06} \cdot d_{16}^{+}=D_{16}^{+} \cdot d_{06} ; D_{07} \cdot d_{17}^{+}=0 \\
& \hat{g}=(1, \Omega ; 0,0,0 ; 0,0)
\end{aligned}
$$

Although this table seems rather long, the imposition of the $\hat{g}$-transformed Killing vector on the previously obtained profiles (16) eliminates most of the above combinations. The only non-trivial ones together with the corresponding relative transformations $\hat{g}$ are

$$
\begin{array}{rl}
3+6 & f=h_{0}+\lambda \rho^{2}, \\
X_{3} & =\left(0, \omega_{1} ; 0,0,0 ; 0,0\right) \quad \hat{g}=i d, \\
X_{6} & =\left(0,0 ; D_{0}, 0, \lambda D_{0} ; 0,0\right), \\
4+7 f & =h_{0} \exp \frac{1}{D_{0}^{2}}\left(\left(\tilde{D}_{0} x\right) \frac{D_{0} \cdot \Omega D_{07}}{\tilde{D}_{0} \Omega \Omega D_{07}}-D_{0} x\right), \\
X_{4} & =\left(1,0 ; D_{0}, 0,0 ; 0,0\right) \quad \hat{g}=\Omega, \\
X_{7} & =\left(0,0 ; \Omega D_{07}, 0,0 ; 0,0\right), \\
6+7 f & =h_{0}+\frac{1}{\left(D_{0}^{2}\right)^{2}}\left[\frac{\left(D_{1}^{+} \tilde{D}_{0}\right)^{2}}{D_{1}^{+} D_{0}}\left(\tilde{D}_{0} x\right)^{2}+\left(D_{1}^{+} D_{0}\right)\left(D_{0} x\right)^{2}+\right. \\
X_{6} & =\left(0,0 ; D_{0}, 0, D_{1}^{+} ; 0,0\right) \quad \hat{g}=\Omega, \\
X_{7} & =\left(0,0 ; \Omega D_{07}, 0,0 ; 0,0\right) .
\end{array}
$$

Applying the same procedure to identical orbits, i.e. imposing two vectors belonging to the same orbit, results in 6 combinations. The non-trivial ones are given by

$$
\begin{aligned}
3+\overline{3} f & =h_{0}+\mu \rho^{2} \\
X_{3} & =\left(0, \bar{\omega}_{1} ; 0,0,0 ; 0,0\right) \quad \hat{g}=\left(1, i d ; d_{0}, 0, \mu d_{0} ; 0,0\right) \\
X_{\overline{3}} & =\left(0, \bar{\omega}_{1} ;-\bar{\omega}_{1} d_{0}, 0,-\mu \bar{\omega}_{1} d_{0} ; 0,0\right), \\
4+\overline{4} f & =h_{0} \exp \frac{1}{D_{0}^{2}}\left[\frac{D_{0}\left(\Omega \bar{D}_{0}-D_{0}\right)}{\bar{D}_{0} \Omega \bar{D}_{0}}\right] \\
X_{4} & =\left(1,0 ; D_{0}, 0,0 ; 0,0\right) \quad \hat{g}=\Omega \\
X_{\overline{4}} & =\left(1,0 ; \Omega \bar{D}_{0}, 0 ; 0,0\right),
\end{aligned}
$$




$$
\begin{gathered}
6+\overline{6} f=h_{0}+\frac{1}{\left(D_{0}^{2}\right)^{2}}\left[\frac{-\left(D_{1}^{+} \tilde{D}_{0}\right)+D_{0}^{2}\left(\tilde{D}_{0} \Omega \bar{D}_{1}^{+}\right)}{\Omega \bar{D}_{0} \bar{D}_{0}}\left(\tilde{D}_{0} x\right)^{2}+\left(D_{1}^{+} D_{0}\right)\left(D_{0} x\right)^{2}+\right. \\
\left.2\left(D_{1}^{+} \tilde{D}_{0}\right)\left(D_{0} x\right)\left(\tilde{D}_{0} x\right)\right], \\
X_{6}=\left(0,0 ; D_{0}, 0, D_{1}^{+} ; 0,0\right) \quad \hat{g}=\Omega \quad D_{1}^{+} \cdot \Omega \bar{D}_{0}=D_{0} \cdot \Omega \bar{D}_{1}^{+}, \\
X_{\overline{6}}=\left(0,0 ; \Omega \bar{D}_{0}, 0, \Omega \bar{D}_{1}^{+} ; 0,0\right) .
\end{gathered}
$$

This completes the classification for impulsive waves with a five-parametric Killing algebra. It is however possible to recast them in a simpler form.

$$
\begin{aligned}
& 3+\overline{3} f=h_{0}+\mu \rho^{2} \text {, } \\
& 3+6 f=h_{0}+\lambda \rho^{2} \text {, } \\
& X_{3}=\left(0, \bar{\omega}_{1} ; 0,0,0 ; 0,0\right), \\
& X_{3}=\left(0, \omega_{1} ; 0,0,0 ; 0,0\right), \\
& X_{6}=\left(0,0 ; D_{0}, 0, \lambda D_{0} ; 0,0\right) \text {, } \\
& 4+7 f=h_{0} \exp \left(-\frac{\Omega \tilde{D}_{07} \cdot x}{D_{07}^{2} \lambda}\right) \text {, } \\
& \bar{X}_{4}=\left(1,0 ; \lambda \Omega \tilde{D}_{07}, 0,0 ; 0,0\right) \\
& X_{7}=\left(0,0 ; \Omega D_{07}, 0,0 ; 0,0\right) \text {, } \\
& X_{6}=\left(0, \bar{\omega}_{1} ; E_{0}, 0, \mu E_{0} ; 0,0\right) \\
& E_{0}=-\bar{\omega}_{1} d_{0} \text {, } \\
& 4+\overline{4} f=h_{0} \exp \left(-\frac{\tilde{E}_{0} \cdot x}{D_{0} \tilde{E}_{0}}\right), \\
& \bar{X}_{4}=\left(1,0 ; \frac{D_{0} \cdot E_{0}}{E_{0}^{2}} \tilde{E}_{0}, 0,0 ; 0,0\right) \\
& \bar{X}_{\overline{4}}=\left(0,0 ; E_{0}, 0,0 ; 0,0\right) \\
& E_{0}=\Omega \bar{D}_{0}-D_{0} \text {, } \\
& 6+7 f=h_{0}+\lambda \frac{\left(D_{1}^{+} x\right)^{2}}{\left(D_{1}^{+}\right)^{2}} \text {, } \\
& \bar{X}_{6}=\left(0,0 ; D_{1}^{+}, 0, \lambda D_{1}^{+} ; 0,0\right) \\
& X_{7}=\left(0,0 ; \tilde{D}_{1}^{+}, 0,0 ; 0,0\right) \text {, } \\
& 6+\overline{6} f(x)=h_{0}+ \\
& \frac{1}{E_{0}^{2}}\left[\lambda\left(E_{0} x\right)^{2}+\bar{\lambda}\left(\tilde{E}_{0} x\right)^{2}\right] \\
& \bar{X}_{6}=\left(0,0 ; E_{0}, 0, \lambda E_{0} ; 0,0\right) \\
& \bar{X}_{\overline{6}}=\left(0,0 ; \tilde{E}_{0}, 0, \bar{\lambda} \tilde{E}_{0} ; 0,0\right) .
\end{aligned}
$$

In order to obtain the above simplified form, we linearly combined those Killing vectors obtained directly from the classification process. The simplification of $6+\overline{6}$ arises from noting that this class actually represents a generic quadratic profile $f(x)=x \cdot M x$, which may be diagonalized giving rise to the eigenvalues $\lambda, \bar{\lambda}$. It is interesting to note that the combination of elements belonging to the same orbit is equivalent to the combination of different ones. So we see that there are actually only three different classes containing 5 Killing vectors. Moreover, the Killing group of $3+\overline{3}$ is actually six-dimensional due to the independence of the profile from $E_{0}$.

Proceeding along the same lines for the combination of three orbits, we find that there are no nontrivial cases. Let us summarize the results in the following table 


\begin{tabular}{|c|c|c|c|}
\hline profile & Killing vectors & $\mathrm{r}$ & type \\
\hline$f=f(x)$ & $\begin{array}{l}\xi_{1}=\partial_{v}, \\
\xi_{2}=2 x \partial_{v}+u \partial_{x}, \\
\xi_{3}=2 y \partial_{v}+u \partial_{y}\end{array}$ & $\overline{3}$ & abelian \\
\hline$f=h(\rho) e^{\frac{1}{\gamma_{1} \phi}}$ & $\begin{array}{l}\xi_{1}, \xi_{2}, \xi_{3}, \\
\xi_{4}=u \partial_{u}-v \partial_{v}-\gamma_{1} \partial_{\phi}\end{array}$ & 4 & $\begin{array}{l}{\left[\xi_{4}, \xi_{1}\right]=\xi_{1},} \\
{\left[\xi_{4}, \xi_{2}\right]=\xi_{2}+\gamma_{1} \xi_{3},} \\
{\left[\xi_{4}, \xi_{3}\right]=\xi_{3}-\gamma_{1} \xi_{2}}\end{array}$ \\
\hline$f=h(\rho)-\frac{2}{\gamma_{1}} \phi$ & $\begin{array}{l}\xi_{1}, \xi_{2}, \xi_{3}, \\
\xi_{4}=-\gamma_{1} \partial_{\phi}+2 \theta(u) \partial_{v}\end{array}$ & 4 & $\begin{array}{l}{\left[\xi_{4}, \xi_{2}\right]=\gamma_{1} \xi_{3}} \\
{\left[\xi_{4}, \xi_{3}\right]=-\gamma_{1} \xi_{2}}\end{array}$ \\
\hline$f=h(\rho)$ & $\begin{array}{l}\xi_{1}, \xi_{2}, \xi_{3} \\
\xi_{4}=\partial_{\phi}\end{array}$ & 4 & $\begin{array}{l}{\left[\xi_{4}, \xi_{2}\right]=-\xi_{3}} \\
{\left[\xi_{4}, \xi_{3}\right]=\xi_{2}}\end{array}$ \\
\hline$f=h(y) e^{-\frac{1}{\lambda} x}$ & $\begin{array}{l}\xi_{1}, \xi_{2}, \xi_{3}, \\
\xi_{4}=u \partial_{u}-v \partial_{v}+\lambda \partial_{x}\end{array}$ & 4 & $\begin{array}{l}{\left[\xi_{4}, \xi_{1}\right]=\xi_{1},} \\
{\left[\xi_{4}, \xi_{2}\right]=\xi_{2}+2 \lambda \xi_{1},} \\
{\left[\xi_{4}, \xi_{3}\right]=\xi_{3}}\end{array}$ \\
\hline$f=h(y)+\left(\lambda x^{2}+2 \bar{\lambda} x y\right)$ & $\begin{array}{l}\xi_{1}, \xi_{2}, \xi_{3} \\
\xi_{4}=\partial_{x}+\theta(u)\left(\lambda \xi_{2}+\bar{\lambda} \xi_{3}\right)\end{array}$ & 4 & {$\left[\xi_{4}, \xi_{2}\right]=2 \xi_{1}$} \\
\hline$f=h(y)$ & $\begin{array}{l}\xi_{1}, \xi_{2}, \xi_{3} \\
\xi_{4}=\partial_{x}\end{array}$ & 4 & {$\left[\xi_{4}, \xi_{2}\right]=2 \xi_{1}$} \\
\hline$f=h_{0}+\lambda \rho^{2}$ & $\begin{array}{l}\xi_{1}, \xi_{2}, \xi_{3} \\
\xi_{4}=\partial_{x}+\lambda \theta(u) \xi_{2} \\
\xi_{5}=\partial_{y}+\lambda \theta(u) \xi_{3} \\
\xi_{6}=\partial_{\phi}\end{array}$ & 6 & $\begin{array}{l}{\left[\xi_{4}, \xi_{2}\right]=2 \xi_{1}} \\
{\left[\xi_{4}, \xi_{6}\right]=\lambda \theta(u) \xi_{3}} \\
{\left[\xi_{5}, \xi_{3}\right]=2 \xi_{1}} \\
{\left[\xi_{5}, \xi_{6}\right]=-\lambda \theta(u) \xi_{2}} \\
{\left[\xi_{6}, \xi_{2}\right]=-\xi_{3}} \\
{\left[\xi_{6}, \xi_{3}\right]=\xi_{2}}\end{array}$ \\
\hline$f=h_{0} e^{-\frac{1}{\lambda} x}$ & $\begin{array}{l}\xi_{1}, \xi_{2}, \xi_{3} \\
\xi_{4}=u \partial_{u}-v \partial_{v}+\lambda \partial_{x} \\
\xi_{5}=\partial_{y}\end{array}$ & 5 & $\begin{array}{l}{\left[\xi_{4}, \xi_{1}\right]=\xi_{1},} \\
{\left[\xi_{4}, \xi_{2}\right]=\xi_{2}+2 \lambda \xi_{1},} \\
{\left[\xi_{4}, \xi_{3}\right]=\xi_{3},} \\
{\left[\xi_{5}, \xi_{3}\right]=2 \xi_{1}}\end{array}$ \\
\hline$f=h_{0}+\left(\lambda x^{2}+\bar{\lambda} y^{2}\right)$ & $\begin{array}{l}\xi_{1}, \xi_{2}, \xi_{3}, \\
\xi_{4}=\partial_{x}+\lambda \theta(u) \xi_{2}, \\
\xi_{5}=\partial_{y}+\bar{\lambda} \theta(u) \xi_{3}\end{array}$ & 5 & $\begin{array}{l}{\left[\xi_{4}, \xi_{2}\right]=2 \xi_{1}} \\
{\left[\xi_{5}, \xi_{3}\right]=2 \xi_{1}}\end{array}$ \\
\hline
\end{tabular}

Let us remark that the above table lists only the non-vanishing commutators and that the profile is not required to obey any field-equations. Specifically the vacuum equations require $f$ to be harmonic. 


\section{4) Rosen form and differentiability conditions}

This section is devoted to the so-called Rosen form of the metric (11). It amounts in the elimination of the $\delta$-term and brings the metric into a continuous form, however, at the price of a discontinuous profile-dependent coordinate transformation. At first sight this procedure might seem somewhat paradoxical since the metric (II) is clearly discontinuous. However, one has to keep in mind that the discontinuous change of coordinates alters the differentiable structure. With respect to the new differentiable structure, i.e. forgetting where the coordinates came from, the metric is $C^{0}$. The explicit form of the transformation is given by

$$
\begin{aligned}
u & =U, \\
v & =V+\theta(U) f(X)+U \theta(U) \frac{1}{4}(\partial f)^{2}, \\
x & =X+U \theta(U) \frac{1}{2} \partial f .
\end{aligned}
$$

Applying this transformation to (11) leaves us with

$$
d s^{2}=-d U d V+\left(\delta_{i j}+\frac{1}{2} U \theta(U) \partial_{i} \partial_{j} f\right)^{2} d X^{i} d X^{j},
$$

which is obviously $C^{0}$. Since it is not possible to explicitly invert (17) for a general profile $f$ let us illustrate the above remarks by considering an impulsive plane wave as a specific example. Its profile is given by

$$
f(x)=\frac{1}{E_{0}^{2}}\left(\lambda\left(E_{0} x\right)^{2}+\bar{\lambda}\left(\tilde{E}_{0} x\right)^{2}\right)
$$

Inserting this $f$ into (17) allows us to read off the inverse transformation:

$$
\begin{aligned}
U & =u, \\
V & =v-\frac{1}{E_{0}^{2}} \theta(u)\left(\frac{\lambda}{1+\lambda u}\left(E_{0} x\right)^{2}+\frac{\bar{\lambda}}{1+\bar{\lambda} u}\left(\tilde{E}_{0} x\right)^{2}\right), \\
X & =x-\frac{1}{E_{0}^{2}} u \theta(u)\left(\frac{\lambda}{1+\lambda u}\left(E_{0} x\right) E_{0}+\frac{\bar{\lambda}}{1+\bar{\lambda} u}\left(\tilde{E}_{0} x\right) \tilde{E}_{0}\right) .
\end{aligned}
$$

However, the three Killing vectors that were smooth with respect to the original differentiable structure are now non-smooth.

$$
\xi_{1}=\partial_{V}
$$




$$
\begin{aligned}
\xi_{2} & =2 X \partial_{V}+U \partial_{X}-U^{2} \theta(U) \frac{\lambda}{1+\lambda U} \partial_{X} \\
\xi_{3} & =2 Y \partial_{V}+U \partial_{Y}-U^{2} \theta(U) \frac{\bar{\lambda}}{1+\bar{\lambda} U} \partial_{Y}
\end{aligned}
$$

Whereas those that were discontinuous now become smooth.

$$
\begin{aligned}
& \xi_{4}=(1+\lambda u \theta(u)) \partial_{x}+2 \lambda \theta(u) x \partial_{v}=\partial_{X} \\
& \xi_{5}=(1+\bar{\lambda} u \theta(u)) \partial_{y}+2 \bar{\lambda} \theta(u) y \partial_{v}=\partial_{Y}
\end{aligned}
$$




\section{Conclusion}

In this work we analyzed the symmetry structure of pp-waves with an impulsive profile (i.e. waves where the curvature is concentrated on a null surface). The mathematical framework describing such an idealized physical situation is necessarily distributional in nature. Therefore an investigation of possible Killing vectors is immediately confronted with the question about the domain of definition of the Killing equation. In general this equation contains products of the Killing vector and the metric which restricts the Killing vector to be $C^{\infty}$. However, due to the specific form of the metric under consideration, it was possible to extend the class of Killing vectors to contain also non-smooth fields while at the same time maintaining a distributionally well-defined structure. This extension lead us to a larger class of symmetries which on physical grounds we think is necessary to include. Consider for instance the plane wave (the last case in our table), which has only three smooth Killing vectors, namely $\xi_{1}, \xi_{2}, \xi_{3}$, while the corresponding non-impulsive wave in the JEK-classification admits a five-parametric isometry group. In the limit to an impulsive wave one exactly recovers the two "missing" Killing vectors $\xi_{4}$ and $\xi_{5}$ as non-smooth symmetries. On the other hand it is in general not possible to obtain the isometry group by taking the limit from a pp-wave with a non-impulsive profile. This is precisely the reason why the JEK-classification fails and additional symmetries show up. One may however ask if those spacetimes which cannot be obtained from boosting a sandwich-wave are physically meaningful. We would like to give an affirmative answer, e.g. the second case in our table, as pointed out in [1] becomes in the vacuum case the so-called AS-metric, whose symmetry-group can be obtained by boosting the Killing vectors of the Schwarzschild-geometry to the velocity of light [7].

Finally we addressed the question of the so-called Rosen-coordinates with respect to which the metric becomes $C^{0}$. The corresponding change of coordinates is of course discontinuous and turns Killing vectors that were smooth with respect to the original differentiable structure into non-smooth vector fields with respect to the new structure.

A natural extension of our work would be the investigation the geodesicequation for impulsive pp-waves, which faces the same definition problems. It seems, however, that the correct setting for this problem requires more sophisticated techniques [8], which allow to control products of distributions. Work in this direction is currently in progress. 


\section{References}

[1] Aichelburg P and Balasin H, Class. Quantum Grav. 13, 723 (1996).

[2] Jordan P Ehlers J and Kundt W, Akad. Wiss. Lit. (Mainz) Abhandl. Math.-Nat. Kl. 2, 21 (1960).

[3] Gel'fand I M and Shilov G E, Generalized Functions Vol. 1, Academic Press (1964).

[4] Penrose R, General Relativity: Papers in the Honor of J. L. Synge, 101,Clarendon Press, Oxford (1972).

[5] Rosen N, Phys. Z. sowjet. 12366 (1937).

[6] Brinkmann M W, Proc. Nat. Acad. Sci. USA 9, 1 (1923).

[7] Aichelburg P and Balasin H,Class. Quantum Grav. 11, L121 (1994).

[8] Colombeau J F, Multiplication of Distributions LNM 1532, Springer (1992). 